\title{
Direct Evaluation of L-DOPA Actions on Neuronal Activity of Parkinsonian Tissue In Vitro
}

\author{
Víctor Plata, Mariana Duhne, Jesús E. Pérez-Ortega, Janet Barroso-Flores, \\ Elvira Galarraga, and José Bargas
}

\begin{abstract}
División de Neurociencias, Instituto de Fisiología Celular, Universidad Nacional Autónoma de México (UNAM), 04510 México City, DF, Mexico
\end{abstract}

Correspondence should be addressed to José Bargas; jbargas@ifc.unam.mx

Received 17 June 2013; Accepted 18 August 2013

Academic Editor: Olivier Darbin

Copyright (C) 2013 Víctor Plata et al. This is an open access article distributed under the Creative Commons Attribution License, which permits unrestricted use, distribution, and reproduction in any medium, provided the original work is properly cited.

\begin{abstract}
Physiological and biochemical experiments in vivo and in vitro have explored striatal receptor signaling and neuronal excitability to posit pathophysiological models of Parkinson's disease. However, when therapeutic approaches, such as dopamine agonists, need to be evaluated, behavioral tests using animal models of Parkinson's disease are employed. To our knowledge, recordings of population neuronal activity in vitro to assess anti-Parkinsonian drugs and the correlation of circuit dynamics with disease state have only recently been attempted. We have shown that Parkinsonian pathological activity of neuronal striatal circuits can be characterized in in vitro cerebral tissue. Here, we show that calcium imaging techniques, capable of recording dozens of neurons simultaneously with single-cell resolution, can be extended to assess the action of therapeutic drugs. We used L-DOPA as a prototypical antiParkinsonian drug to show the efficiency of this proposed bioassay. In a rodent model of early Parkinson's disease, Parkinsonian neuronal activity can be returned to control levels by the bath addition of L-DOPA in a reversible way. This result raises the possibility to use calcium imaging techniques to measure, quantitatively, the actions of anti-Parkinsonian drugs over time and to obtain correlations with disease evolution and behavior
\end{abstract}

\section{Introduction}

Idiopathic Parkinson's disease is a progressive incapacitating movement disorder whose treatment remains unsatisfactory. Behavioral tests are commonly used in Parkinsonian animal models to assess experimental therapeutic approaches and drugs intended to be helpful in Parkinsonian patients [1]. Nevertheless, after half a century, L-DOPA remains the treatment of choice and the "gold standard" [2]. However, experimental paradigms to efficiently assess new anti-Parkinsonian drugs and treatments using in vitro brain tissue, during neuronal activity, are lacking.

Recently, we used calcium imaging techniques to record dozens of neurons simultaneously with single-cell resolution to prove that the activity of the striatal microcircuit recorded in vitro during dopamine (DA) depletion is radically different than that recorded in the controls [3]. As previously reported, the Parkinsonian circuit had increased synaptic and neuronal activity [4-10] and was particularly engaged into a dominant network state [3] due to increased neuronal synchronization
$[3,11-13]$. The action of a DA $\mathrm{D}_{1}$-receptor agonist in this circuit dissolved the dominant state but did not reverse the enhanced activity [3]. In contrast, it has been shown that the use of DA $\mathrm{D}_{2}$-receptor agonists appears to better restore the balance between direct and indirect pathways activity [14], suggesting that $\mathrm{D}_{2}$-receptor agonists may be better therapeutic agents. However, this latter action may be indirect, since inhibitory connections are stronger from indirect to direct pathway neurons $[15,16]$ and $\mathrm{D}_{2}$-receptor agonists may also inhibit these synapses [17], thus, suppressing their braking effect on direct neurons excitability. In addition, previous work has also stated that the synergistic activation of both $\mathrm{D}_{1}$ and $\mathrm{D}_{2}$-receptor agonists may be necessary to have a better amelioration of signs and to delay dyskinesias [18-21], a good reason why L-DOPA remains as the "gold standard" [2].

Due to this controversy, we asked whether an in vitro bioassay can be designed to test the actions of drugs directly onto neuronal activity. Therefore, the goals of the present report are as follows: first, to test the actions of L-DOPA directly on brain tissue maintained in vitro (corticostriatal 
brain slice preparation); second, to observe the actions of L-DOPA on the simultaneous activity of dozens of neurons in the DA-depleted striatal microcircuit. In particular, we wanted to see L-DOPA actions at a stage considered as early Parkinsonian and determine whether it can dissolve the dominant network state [3]. Finally, we want to see, in the present preparation, whether a measure of global population activity may be enough to compare different drugs and open the path to perform, in the future, concentration-response functions.

The present work shows that, in the early Parkinsonian state, L-DOPA restores neuronal activity to control levels. This action was readily reversible. Therefore, the present prototypical experimental design is open to develop more sophisticated network analyses to expose finer differences between therapeutic agents $[22,23]$, since single-cell resolution is maintained. To conclude, the present preparation deserves further exploration in order to validate a bioassay to investigate and compare the actions of anti-Parkinsonian agents quantitatively.

\section{Material and Methods}

2.1. Slice Preparation. Male mice or rats were housed in clear plastic cages and maintained on a stable 12:12 hours dark/light cycle at room temperature $\left(22^{\circ} \mathrm{C}\right)$, with food and water ad libitum in our Animal House. The number of animals used in the experimental samples was the minimal possible to attain statistical significance. All the procedures followed the National University of Mexico guidelines, the Guide for the Care and Use of Laboratory Animals (National Institutes of Health), in accordance with the EC Directive 86/609/EEC for animal experiments. The slice preparation and its analysis have been described before [3, 20, 22]. Briefly, corticostriatal slices $(250 \mu \mathrm{m}$ in thickness) were obtained either from control or 6-hydroxydopamine (6OHDA) treated rodents (mice or rats, postnatal day 23-29).

Because experiments using 6-OHDA treated animals were done mostly in mice, the timing of the protocol in these animals is reported: lesion with 6-OHDA was done at day 21 , turning behavior to check the hemi-Parkinsonian model was done at day 24 , and recording of slices with calcium imaging was done at day 26 or later. Slices were cut in icecold bath saline $\left(4^{\circ} \mathrm{C}\right)$ containing the following (in $\mathrm{mM}$ ): $123 \mathrm{NaCl}, 3.5 \mathrm{KCl}, 1 \mathrm{MgCl}_{2}, 1 \mathrm{CaCl}_{2}, 26 \mathrm{NaHCO}_{3}$, and 11 glucose $\left(25^{\circ} \mathrm{C}\right.$; saturated with $95 \% \mathrm{O}_{2}$ and $5 \% \mathrm{CO}_{2} ; \mathrm{pH}=7.4$; $298 \mathrm{mOsm} / \mathrm{L})$. Slices were then transferred to saline at room temperature $\left(21-25^{\circ} \mathrm{C}\right)$ where they remained for at least $1 \mathrm{~h}$ before recording.

2.2. The 6-OHDA Hemi-Parkinsonian Model. The method to produce the 6-OHDA rodent model of hemi-Parkinsonism, in rats and mice, has been described extensively [3, 24]. Degree of lesion in animals was tested by both tyrosine hydroxylase (TH) immunocytochemistry and turning behavior [24]. The striatum from the injured side was tested one week after the behavioral test.
2.3. Calcium Imaging. Calcium imaging techniques to record, indirectly, the electrical activity of dozens of neurons simultaneously during control conditions and during DA depletion have also been extensively described [3, 20, 22-24]. In this work, we employed $10 \mu \mathrm{M}$ fluo-8 AM (Tef Labs, Austin, TX; with 0.1\% DMSO) and an upright microscope with 10x water immersion objective (E600FN Eclipse, Nikon, Melville, NY). A series of images were acquired with a cooled CCD camera (SenSys 1401E, Roper Scientific, Tucson, AZ) at $100-250 \mathrm{~ms} /$ frame.

Active neurons within the field of view were automatically selected by a program written in LabView (National Ins. Mexico) which also processed the sequence of images. The changes of fluo-8 fluorescence originated from neuronal discharge [22-24] eliciting $\mathrm{Ca}^{2+}$ signals as $\left(F_{i}-F_{o}\right) / F_{o}$, where $F_{i}$ is fluorescence intensity at any frame and $F_{o}$ is resting fluorescence. Duration of neuronal discharge was approximated based on the first time derivative of these $\mathrm{Ca}^{2+}$ signals [22]. Statistical significance used a threshold value given by 2.5 times the standard deviation of the noise. In this way, the neuronal activity over time was graphed in raster plots, where each line in the $y$-axis represents the activity of a single neuron and the $x$-axis represents time in minutes. A histogram shows the summed activity of all neurons (as a field recording) except that each neuron composing a peak of synchronization can be identified. Here, the histogram was used to detect episodes with spontaneous synchronization of several neurons. A horizontal bar indicates the time when LDOPA was added to the bath saline.

2.4. Statistical Methods. Most statistical methods used have been previously described $[22,23]$. To determine the statistical significance of a set of coactive neurons (neuronal vectors), Monte Carlo simulations with 10,000 replications were performed. To analyze the dynamics of the neuronal microcircuit, we employed network states or significant peaks of coactive neurons $[22,23]$. A similarity index was used to determine if these peaks of synchronization contained the same or different neurons. A method for dimensional reduction, locally linear embedding (LLE), was used to project the neuronal vectors in a plane and, thus, follow the dynamics of the circuit [3, 22-24]. To compare global neuronal activity over a given time, we plotted both the cumulative distribution of all cell activity and the cumulative recruitment of neurons. The rates of these cumulative relationships were approximated with ad hoc linear regressions. Their average rates of change (slopes) \pm their estimation errors were compared for significant differences with nonpaired Student's $t$ tests, experiment by experiment. Average significance is reported. In addition, for sample comparisons of these parameters, we used Wilcoxon's $T$ statistic for paired samples and KruskalWallis statistic for nonpaired samples.

2.5. Chemicals. 3,4-Dihydroxy-L-phenylalanine (L-DOPA), 6-hydroxy-dopamine hydrobromide (6-OHDA), and dimethyl sulfoxide (DMSO) were obtained from Sigma-Aldrich (Mexico), Fluo-8-AM and Fluo-8-sodium salt were obtained from AAT Bioquest (Sunnyvale, CA), and ketamine 
hydrochloride and xylazine hydrochloride were obtained from PiSA (Mexico).

\section{Results and Discussion}

As shown in previous work, the striatal circuit appears almost silent in control conditions, with very little activity (Figure 1(a): each point represents the discharge of a neuron and each line denotes the activity of a neuron along time) $[3,8,10,11]$. In contrast, after DA depletion neuronal activity in the striatal circuit is enhanced [3-10] (Figure 1(b)). The histogram of summed activity does not show significant peaks of synchronization in the control (Figure 1(c)) but exhibits spontaneous and significant peaks of neuronal synchronization (network states in color) in the DA-depleted tissue. This finding is in agreement with field potential waves or oscillations recorded electrophysiologically [3-13] ( $n=6$; Figure 1(d)).

The color of synchronization peaks (column neuronal vectors) indicates that the same active neurons make up the peaks (confirmed by the similarity index of the Parkinsonian activity; Figure 1(e): which shows abundance and repetitive activation of a similar patterned mosaic). Dimensional reduction using LLE [22] confirms that most network states contain similar neurons (red), suggesting that circuit dynamics is reduced to the frequent recurrent activity of a dominant network state [3] (red; Figure 1(f)). Figures 1(g) and 1(h) illustrate ways to measure global neuronal activity over a time span to compare control and DA-depleted circuits. Cumulative activity indicates consecutive addition of all bars (active neurons) of activity histograms (Figures 1(c) and 1(d) over time (Figure $1(\mathrm{~g})$ ) in both control (black traces) and the DAdepleted circuit (red traces). The rate of change (tendencies) of cumulative activities can be approximated with ad hoc fitting of straight lines where the rates of cumulative activity are the slopes of the lines \pm their standard estimation errors ( $95 \%$ confidence). In control (Figure 1(g)), rate of cumulative activity was significantly larger for DA-depleted than control tissue (mean rate \pm mean estimation error): $35 \pm 0.12$ act. $/ \mathrm{min}$ in the controls, while it was $105 \pm 0.28$ act./min in the DAdepleted tissue $(n=6 ; P<0.001)$ [3]. Cumulative cell recruitment shows the addition of new neurons activated over time (Figures 1(a) and 1(b) in both control (black traces) and the DA-depleted circuit (red traces; Figure 1(h)). In controls (Figure 1(h)), rate of neuronal recruitment over time was significantly larger for DA-depleted than control tissue (mean rate \pm mean estimation error): $4 \pm 0.26$ cells $/ \mathrm{min}$ in the controls, while it was $9 \pm 0.31$ cells/min in the dopaminedepleted tissue $(n=6 ; P<0.0025)$.

In summary, it is shown that prominent attributes previously reported for the DA-depleted circuit as compared to the controls were fulfilled in the present sample of in vitro experiments. There was more activity in the DA-depleted tissue than in the control [3-10], this increased activity was characterized by the spontaneous synchronization of neurons, and because these neurons conformed similar peaks of synchronization, we conclude that they are mostly caused by the recurrent activity of the same network state [3].
Figure 2(a) shows the activity of over 70 neurons in DAdepleted tissue, and the histogram in Figure 2(b) shows the summed activity of all neurons [22]. Colors indicate neuronal column vectors or peaks of synchronization as indicated by the similarity index. Because the animals are hemiParkinsonian after a lesion with 6-OHDA (see Section 2), first two frames show that the circuit activity is higher than in control animals (DA depleted, cf. Figure 1(a)). This increased pathological activity is the control in the present experiment. Next three frames in Figure 1(a) show the activity of the DAdepleted circuit during the addition of $1 \mu \mathrm{M} \mathrm{L}$-DOPA to the bath saline (bar). Note that L-DOPA gradually eliminated peaks of coactive cells and virtually restores control resting activity. Washing off L-DOPA restored Parkinsonian activity (last two frames), showing that actions of the drug were reversible.

Note that the rate of cumulative activity (Figure 2(c)) decreased after addition of $1 \mu \mathrm{M}$ L-DOPA to the bath saline, from (mean rate \pm mean estimation error) $105 \pm 0.28$ act. $/ \mathrm{min}$ (DA-depleted tissue) to $24 \pm 0.15(n=6 ; P<0.001)$. The rate of cell recruitment over time also decreased from $9 \pm 0.31$ to $3 \pm 0.25(P<0.001)$. The changes are clear at a glance and samples are summarized with box Tukey distributions (medians and percentiles) in Figures 2(e) and 2(f). The DA-depleted sample and the sample with L-DOPA administration employed the same tissue (since striatal tissue from 6-OHDA injured animals was used for controls and the test sample used the same tissue in the presence of LDOPA). Therefore, parameters significance between these samples was tested with a Wilcoxon's $T$ paired statistic $(P<0.028)$. Control sample was taken from different animals, and there were no significant differences between its parameters and those from the L-DOPA sample, suggesting that during early stages of the Parkinsonian state, L-DOPA is capable of restoring neuronal activity to levels that are undistinguishable from those in the controls. However, there were significant differences between control and DA-depleted samples (Kruskal-Wallis, $P<0.05$ ).

It is shown that calcium imaging of neuronal populations allows direct observation and measurement of the actions of anti-Parkinsonian drugs on the dopamine-depleted striatal microcircuit. We used the gold standard of anti-Parkinsonian therapeutics: L-DOPA [2], in order to compare its actions with other drugs tested in the future (in use or novel). Although graphically, raster plots and activity histograms show the differences between control neuronal activity and enhanced pathological activity observed in DA-depleted circuits [3-13], and the later, with activity seen after addition of L-DOPA, we show that cumulative activity and cumulative recruitment of neurons offer quantitative measurements and ranges capable of allowing concentration-response plots in future studies. L-DOPA significantly reduced the enhanced pathological activity of the DA-depleted circuit. In addition, we show that L-DOPA actions can be readily reversed upon washing off [13]. Therefore, the use of this preparation may also be used to shed light on the development of L-DOPA induced receptor hypersensitivity and later dyskinesia in animal models [19, 21]. Moreover, the combination of selective dopamine receptor ligands with transgenic mice would allow 


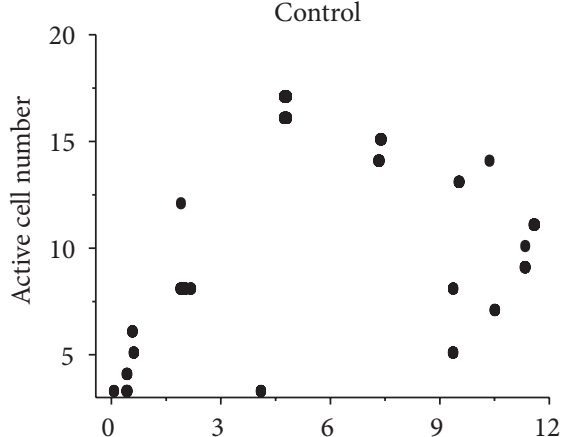

(a)

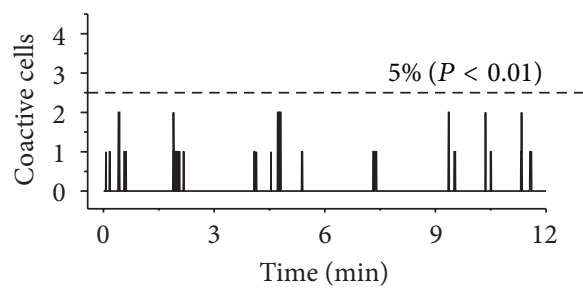

(c)

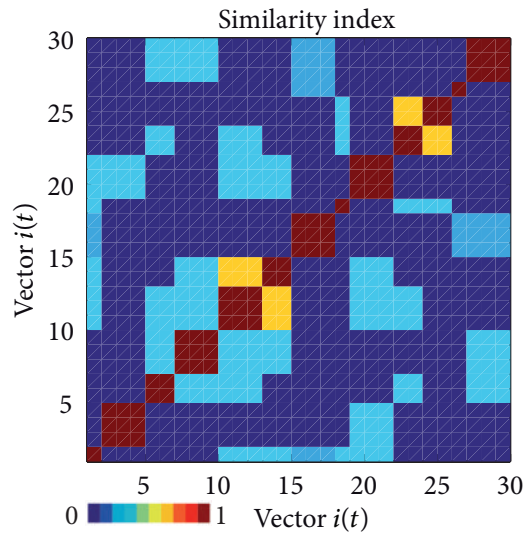

(e)

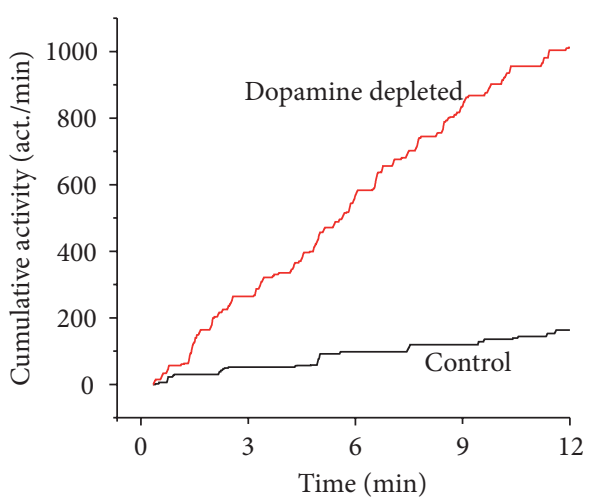

(g)

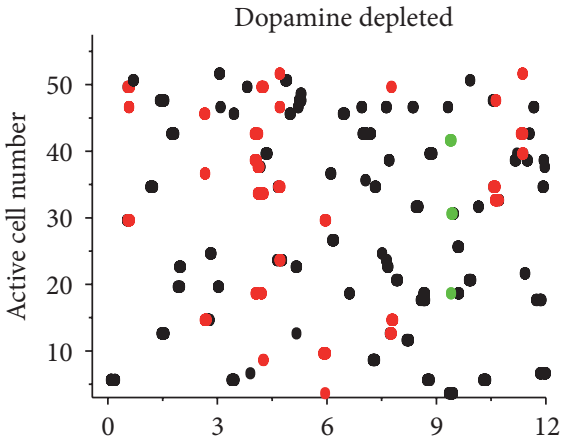

(b)

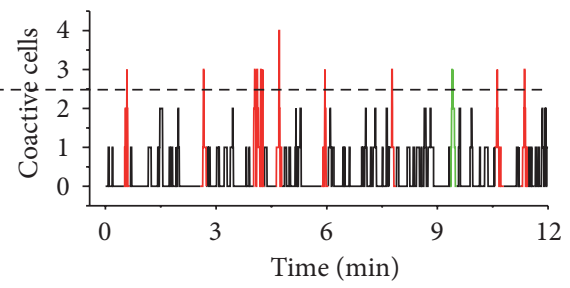

(d)

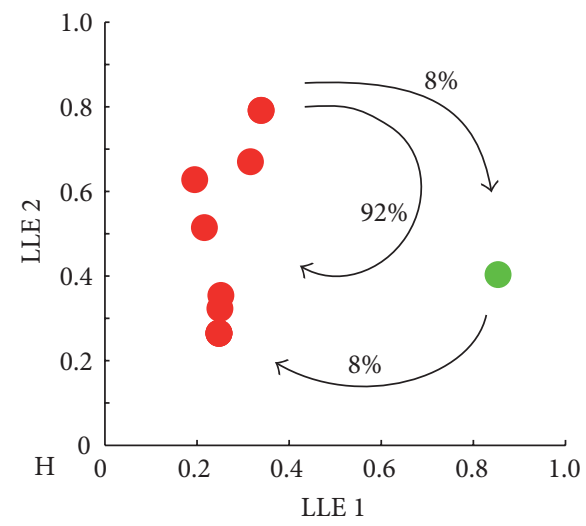

(f)

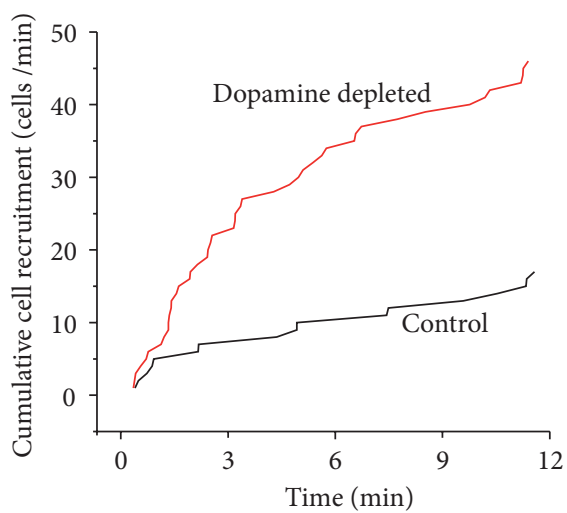

(h)

FIGURE 1: Control and dopamine-depleted striatal microcircuits. (a), (b) Raster plots showing spontaneous neuronal activity in striatal microcircuits in control (a) and after dopamine (DA) depletion (b). Note, increased activity in the DA-depleted circuit. (c), (d) Histograms of summed activity (columns) shown in raster plots frame by frame. Only the DA-depleted microcircuit had significant peaks of coactive neurons: peaks of synchronization of the same color indicate that similar neurons discharged in those instances. (e) Similarity indices of all neuronal vectors representing network states as a function of time in the DA-depleted circuit. (f) Multidimensional reduction of vectors representing network states using locally linear embedding (LLE). Note that one neuronal vector (red: projections of the peaks of synchronization above) activates more frequently creating a dominant network state that absorbs most active neurons. (g), (h) Cumulative activity and cumulative cell recruitment in both control (black traces) and DA-depleted circuit (red traces). 


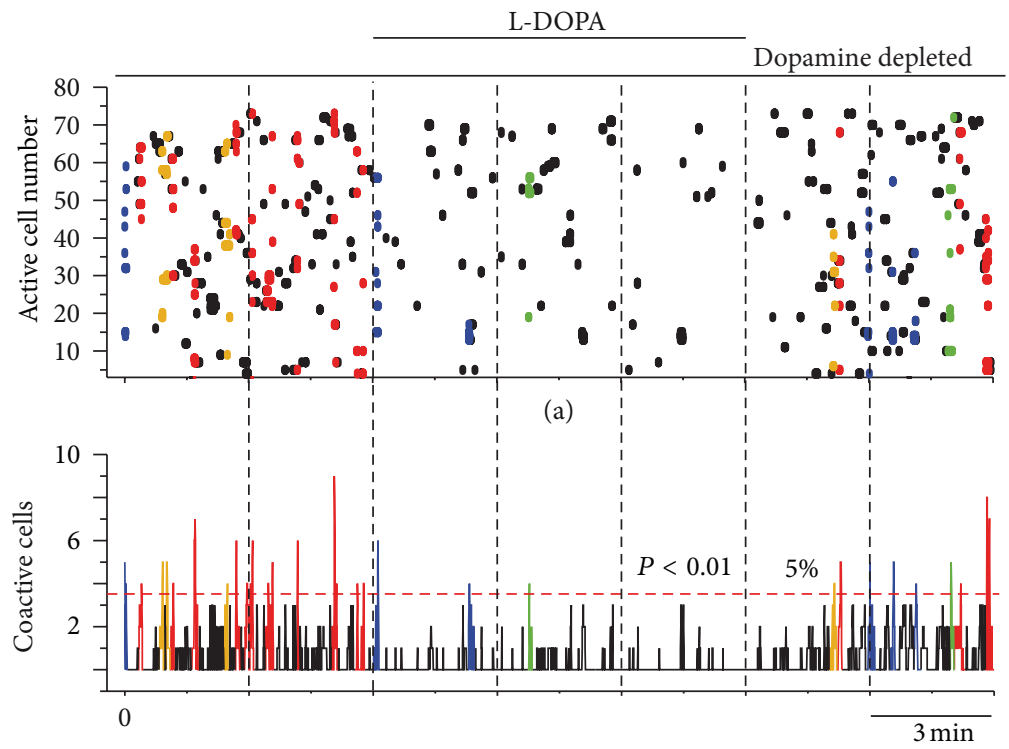

(b)

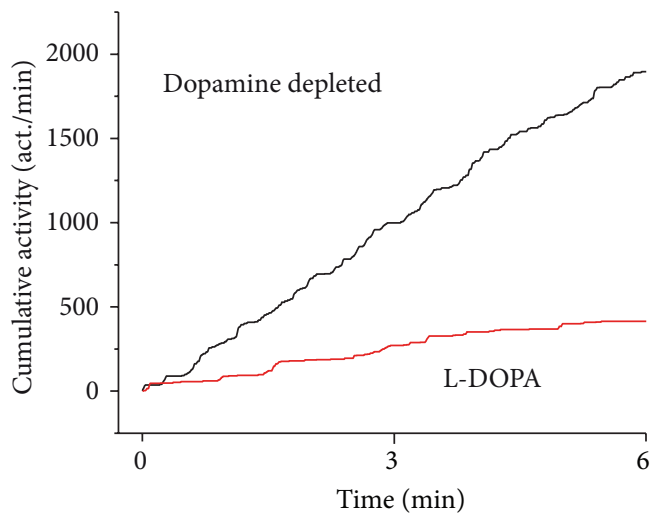

(c)

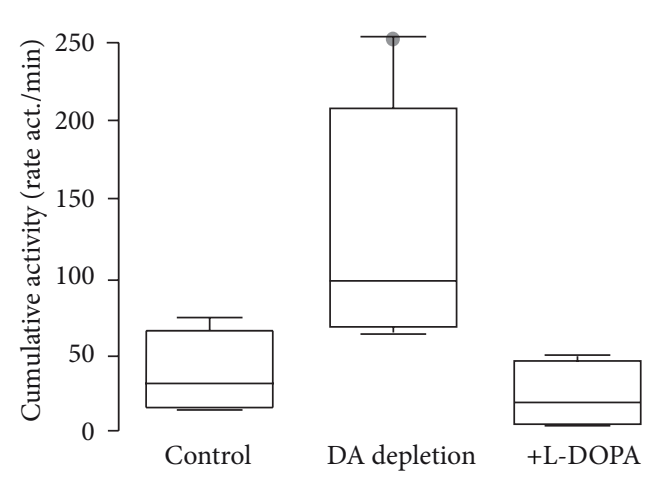

(e)

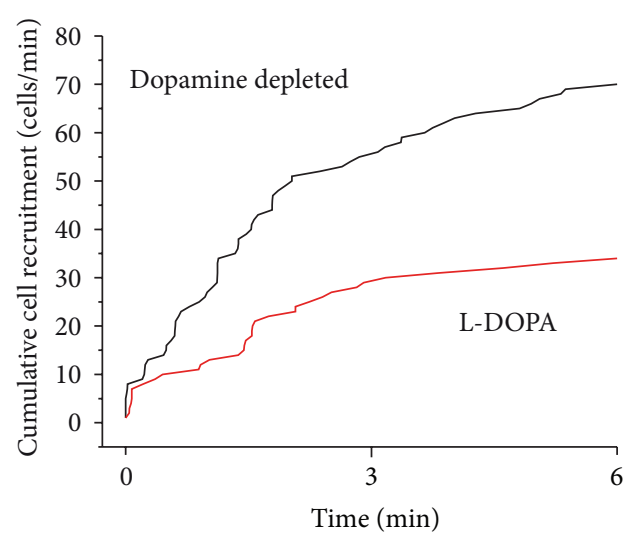

(d)

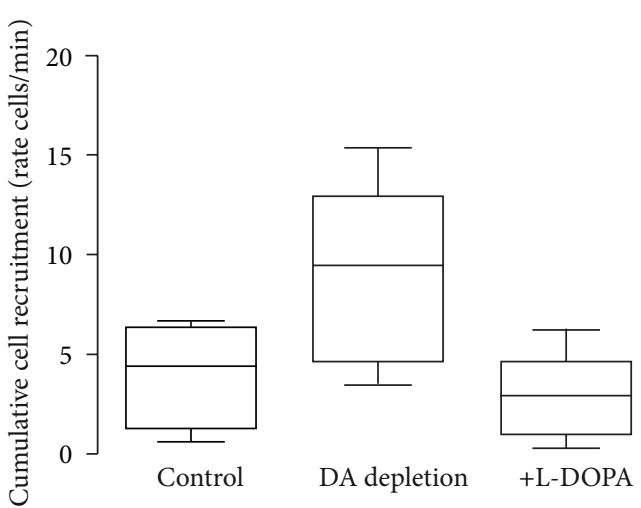

(f)

FIGURE 2: Actions of L-DOPA on DA-depleted striatal microcircuit. (a) Raster plot showing neuronal activity before and during the administration of $1 \mu \mathrm{M} \mathrm{L-DOPA}$ into the bath saline (black bar). Note that neuronal activity is reduced during L-DOPA. L-DOPA actions are reversible. (b) Summed neuronal activity histogram: abundant peaks of neurons firing in synchrony are present before and after but not during L-DOPA present in the bath saline. (c), (d) Cumulative activity and cumulative cell recruitment in the DA-depleted microcircuit both before (black traces) and during L-DOPA administration (red traces). (e), (f) Tukey box plots showing samples distributions of cumulative activity and cumulative cell recruitment, respectively. Neuronal activity is significantly different when one compares DA-depletion versus either control $(P<0.05)$ or L-DOPA $(P<0.028)$. Difference between control and L-DOPA samples is not significant. 
the testing of the different models of Parkinsonism $[12,14,18$, 19].

In addition to enhanced activity in the DA-depleted microcircuit, the capability of having single-cell resolution allows to see the formation of coactive neuron sets or network states $[20,22,23]$. The analysis of network states shows the similarity between peaks of synchronization and shows that microcircuit dynamics (with LLE) is basically dependent on the recurrence of the same dominant network state that absorbs most active neurons [3]. This dynamics interrupts normal alternation between network states as occurring in active control microcircuits $[20,22,23]$ and may be the cellular correlate of the increased oscillatory activity found in Parkinsonian subjects [8, 9, 11-13]. Interestingly, L-DOPA was capable of dissolving this dominant state in this stage of the disease and returning neuronal activity to control levels. In contrast, a $\mathrm{D}_{1}$-like receptor agonist could dissolve the dominant state but did not reduce enhanced pathological activity [3]. $\mathrm{D}_{2}$-like receptor agonists apparently decrease pathological activity [14] but do not avoid $\mathrm{D}_{1}$-like receptor hypersensitivity resulting in pathological states [19, 21]. A battery of $\mathrm{D}_{2}$-like receptor agonists may need to be tested with the present technique. Apparently, combinations of both agonists [18, 20, 24] with coadjutant agonists from other receptors present in striatal neurons may allow an increased understanding of anti-Parkinsonian therapeutics.

\section{Conclusions}

Calcium imaging of populations of striatal neurons with single-cell resolution allows a clear and quantitative observation of anti-Parkinsonian actions of L-DOPA in DA-depleted striatal circuit recorded in vitro, paving the way for future bioassays for drug testing and correlations with disease stage, and even perhaps the achievement of functional biopsies. To reach these goals, the present preparation needs to be compared and correlated with known behavioral bioassays and in different stages of the disease, from early Parkinsonism to dyskinesias and wearing off.

We have previously shown that in other basal ganglia nuclei it is enough to add antagonists for both $\mathrm{D}_{1}$ - and $\mathrm{D}_{2}$ like receptors to the bath saline to induce the oscillatory neuronal activity [25] characteristic of Parkinson's disease. This is harder to see in the striatum, where several neurons need to be synchronized [11-13]. The present preparation, in combination with transgenic animals and other techniques, may allow the study of the extrinsic and intrinsic components that induce pathological activity.

Finally, the present preparation may not only serve to study Parkinson's disease. Correlates between neuronal activity recorded in vitro and multi-unit/field recordings done in vivo may be correlated at the circuit level in many other nuclei and disease states.

\section{Acknowledgments}

The authors thank Antonio Laville, Gabriela X Ayala, Adriana Hernández, and Claudia Rivera for technical support, animal care, and advice. This work was supported by Dirección General de Asuntos del Personal Académico (DGAPA) Universidad Nacional Autónoma de México (UNAM) Grants IN-205610 to José Bargas and IN-206010 to Elvira Galarraga; CONACyT (Mexico) Grants 98004 to Elvira Galarraga and 154131 to José Bargas; and the Mexico-Germany Agreement Consejo Nacional de Ciencia y TecnologíaDeutsche Forschungsgemeinschaft (CONACyT-DFG) Grant I0110/193/10 FON.INST.-29-10 to José Bargas. Víctor Plata had CONACyT doctoral fellowships. Data in this work are part of his doctoral dissertation in the Posgrado en Ciencias Biomédicas de la Universidad Nacional Autónoma de México.

\section{References}

[1] M. Herrera-Marschitz, G. Arbuthnott, and U. Ungerstedt, "The rotational model and microdialysis: significance for dopamine signalling, clinical studies, and beyond," Progress in Neurobiology, vol. 90, no. 2, pp. 176-189, 2010.

[2] N. B. Mercuri and G. Bernardi, “The 'magic' of L-dopa: why is it the gold standard Parkinson's disease therapy?" Trends in Pharmacological Sciences, vol. 26, no. 7, pp. 341-344, 2005.

[3] O. Jáidar, L. Carrillo-Reid, A. Hernández, R. Drucker-Colín, J. Bargas, and A. Hernández-Cruz, "Dynamics of the Parkinsonian striatal microcircuit: entrainment into a dominant network state," Journal of Neuroscience, vol. 30, no. 34, pp. 1132611336, 2010.

[4] C. Oye, R. Bouchard, R. Boucher, and L. J. Poirier, "Spontaneous activity of the putamen after chronic interruption of the dopaminergic pathway: effect of L-dopa," Journal of Pharmacology and Experimental Therapeutics, vol. 175, no. 3, pp. 700-708, 1970.

[5] E. Galarraga, J. Bargas, D. Martinez-Fong, and J. Aceves, "Spontaneous synaptic potentials in dopamine-denervated neostriatal neurons," Neuroscience Letters, vol. 81, no. 3, pp. 351-355, 1987.

[6] P. Calabresi, N. B. Mercuri, G. Sancesario, and G. Bernardi, "Electrophysiology of dopamine-denervated striatal neurons. Implications for Parkinson's disease," Brain, vol. 116, no. 2, pp. 433-452, 1993.

[7] K.-C. Tang, M. J. Low, D. K. Grandy, and D. M. Lovinger, "Dopamine-dependent synaptic plasticity in striatum during in vivo development," Proceedings of the National Academy of Sciences of the United States of America, vol. 98, no. 3, pp. 12551260, 2001.

[8] M.-T. Chen, M. Morales, D. J. Woodward, B. J. Hoffer, and P. H. Janak, "In vivo extracellular recording of striatal neurons in the awake rat following unilateral 6-hydroxydopamine lesions," Experimental Neurology, vol. 171, no. 1, pp. 72-83, 2001.

[9] K. Y. Tseng, F. Kasanetz, L. Kargieman, L. A. Riquelme, and M. G. Murer, "Cortical slow oscillatory activity is reflected in the membrane potential and spike trains of striatal neurons in rats with chronic nigrostriatal lesions," Journal of Neuroscience, vol. 21, no. 16, pp. 6430-6439, 2001.

[10] L. Liang, M. R. DeLong, and S. M. Papa, "Inversion of dopamine responses in striatal medium spiny neurons and involuntary movements," Journal of Neuroscience, vol. 28, no. 30, pp. 75377547, 2008.

[11] A. Raz, A. Feingold, V. Zelanskaya, E. Vaadia, and H. Bergman, "Neuronal synchronization of tonically active neurons in 
the striatum of normal and parkinsonian primates," Journal of Neurophysiology, vol. 76, no. 3, pp. 2083-2088, 1996.

[12] J. R. Walters and D. A. Bergstrom, "Basal ganglia network synchronization in animal models of Parkinson's disease," in Cortico-Subcortical Dynamics in Parkinson Disease, pp. 117-142, Contemporary Neuroscience, 2009.

[13] N. Lemaire, L. F. Hernandez, D. Hu, Y. Kubota, M. W. Howe, and A. M. Graybiel, "Effects of dopamine depletion on LFP oscillations in striatum are task- and learning-dependent and selectively reversed by L-DOPA," PNAS, vol. 109, no. 44, pp. 18126-18131, 2012.

[14] B. Ballion, F. Frenois, C. L. Zold, J. Chetrit, M. G. Murer, and F. Gonon, "D2 receptor stimulation, but not D1, restores striatal equilibrium in a rat model of Parkinsonism," Neurobiology of Disease, vol. 35, no. 3, pp. 376-384, 2009.

[15] S. Taverna, E. Ilijic, and D. J. Surmeier, "Recurrent collateral connections of striatal medium spiny neurons are disrupted in models of Parkinson's disease," Journal of Neuroscience, vol. 28, no. 21, pp. 5504-5512, 2008.

[16] V. G. López-Huerta, L. Carrillo-Reid, E. Galarraga et al., “The balance of striatal feedback transmission is disrupted in a model of parkinsonism," Journal of Neuroscience, vol. 33, no. 11, pp. 4964-4975, 2013.

[17] T. Perez-Rosello, A. Figueroa, H. Salgado et al., "Cholinergic control of firing pattern and neurotransmission in rat neostriatal projection neurons: role of $\mathrm{Ca}_{V} 2.1$ and $\mathrm{Ca}_{V} 2.2 \mathrm{Ca}^{2+}$ channels," Journal of Neurophysiology, vol. 93, no. 5, pp. 25072519, 2005.

[18] X.-T. Hu and R. Y. Wang, "Comparison of effects of D-1 and D-2 dopamine receptor agonists on neurons in the rat caudate putamen: an electrophysiological study," Journal of Neuroscience, vol. 8, no. 11, pp. 4340-4348, 1988.

[19] B. Picconi, D. Centonze, K. Håkansson et al., "Loss of bidirectional striatal synaptic plasticity in L-DOPA-induced dyskinesia," Nature Neuroscience, vol. 6, no. 5, pp. 501-506, 2003.

[20] L. Carrillo-Reid, S. Hernández-López, D. Tapia, E. Galarraga, and J. Bargas, "Dopaminergic modulation of the striatal microcircuit: receptor-specific configuration of cell assemblies," Journal of Neuroscience, vol. 31, no. 42, pp. 14972-14983, 2011.

[21] L. Li and F. M. Zhou, "Parallel dopamine D1 receptor activity dependence of L-dopa-induced normal movement and dyskinesia in mice," Neuroscience, vol. 236, pp. 66-76, 2013.

[22] L. Carrillo-Reid, F. Tecuapetla, D. Tapia et al., "Encoding network states by striatal cell assemblies," Journal of Neurophysiology, vol. 99, no. 3, pp. 1435-1450, 2008.

[23] L. Carrillo-Reid, F. Tecuapetla, O. Ibáñez-Sandoval, A. Hernández-Cruz, E. Galarraga, and J. Bargas, "Activation of the cholinergic system endows compositional properties to striatal cell assemblies," Journal of Neurophysiology, vol. 101, no. 2, pp. 737-749, 2009.

[24] V. G. López-Huerta, E. Blanco-Hernández, J. Bargas, and E. Galarraga, "Presynaptic modulation by somatostatin in the rat neostriatum is altered in a model of Parkinsonism," Journal of Neurophysiology, vol. 108, no. 4, pp. 1032-1043, 2012.

[25] J. D. J. Aceves, P. E. Rueda-Orozco, R. Hernández et al., "Dopaminergic presynaptic modulation of nigral afferents: Its role in the generation of recurrent bursting in substantia nigra pars reticulata neurons," Frontiers in Systems Neuroscience, no. 4, article 6, 2011. 

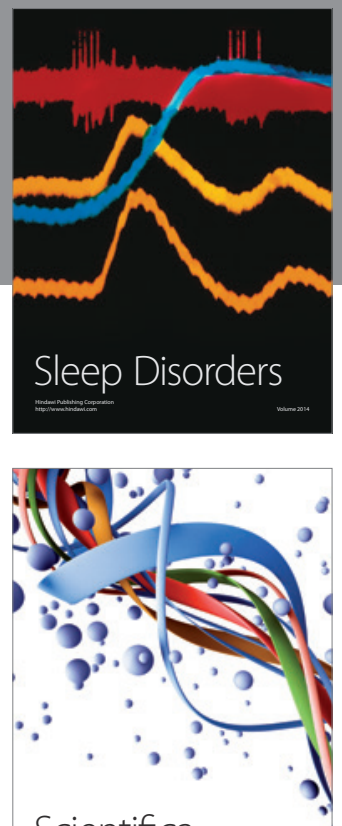

Scientifica
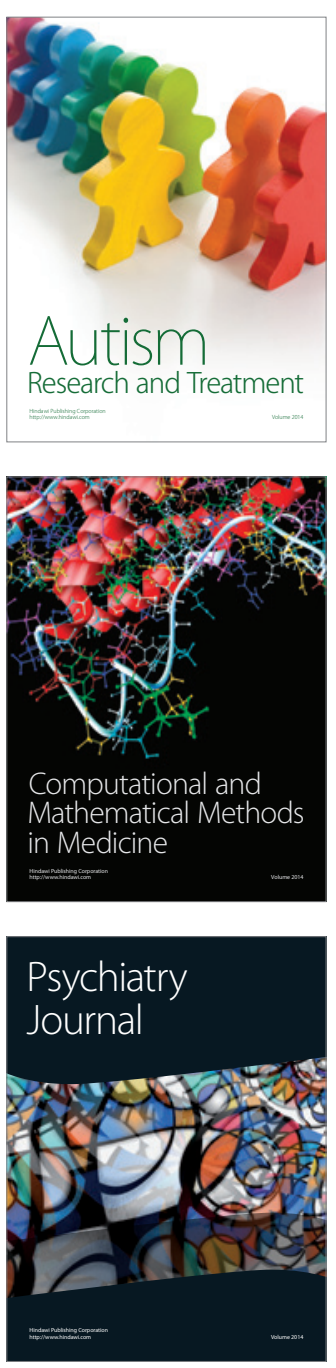
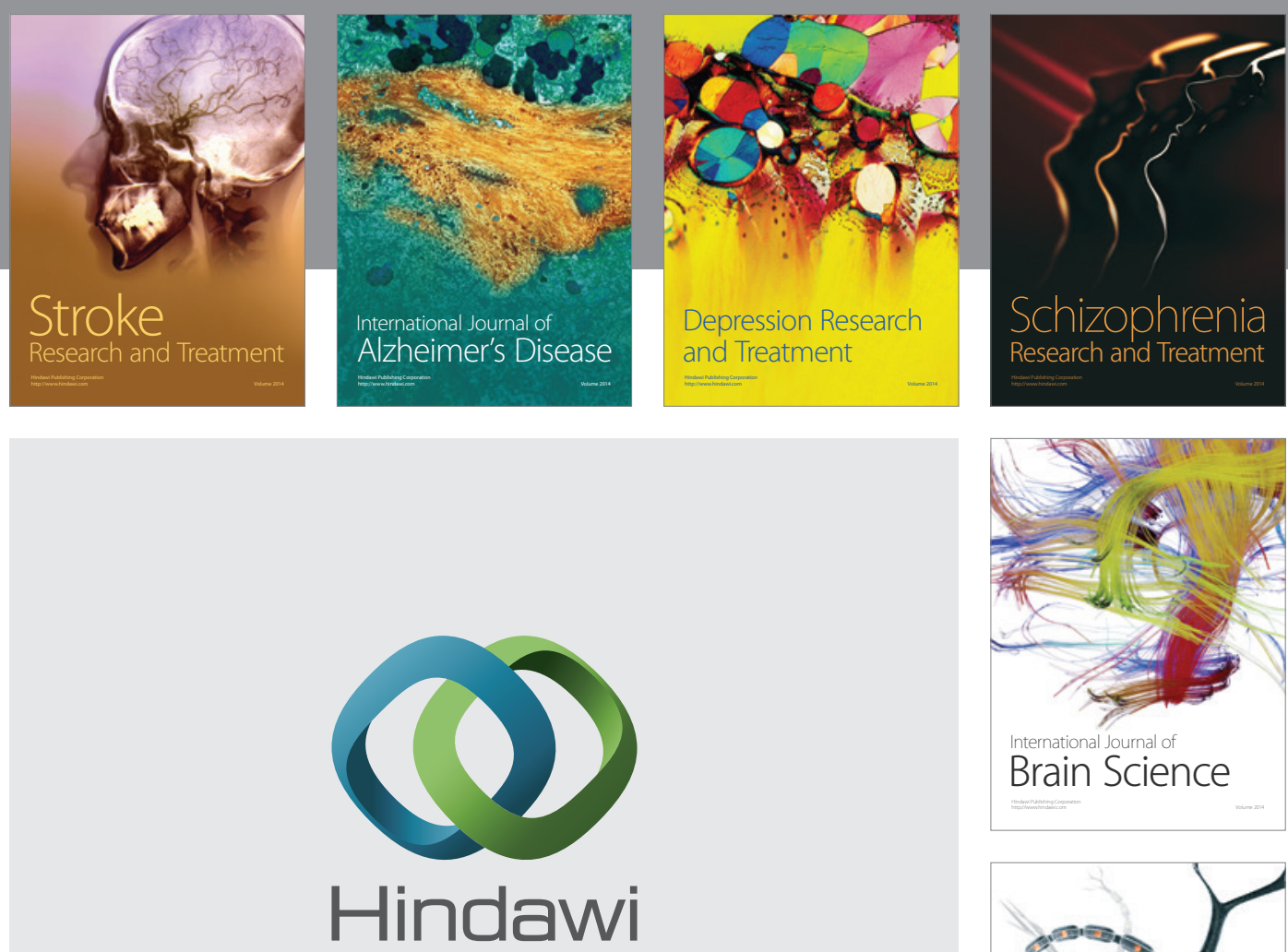

Submit your manuscripts at

http://www.hindawi.com
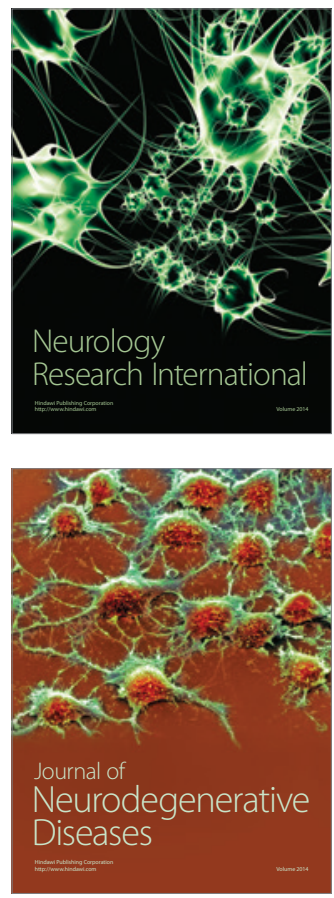

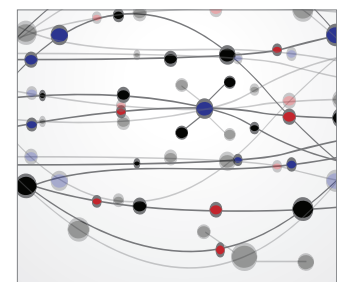

The Scientific World Journal
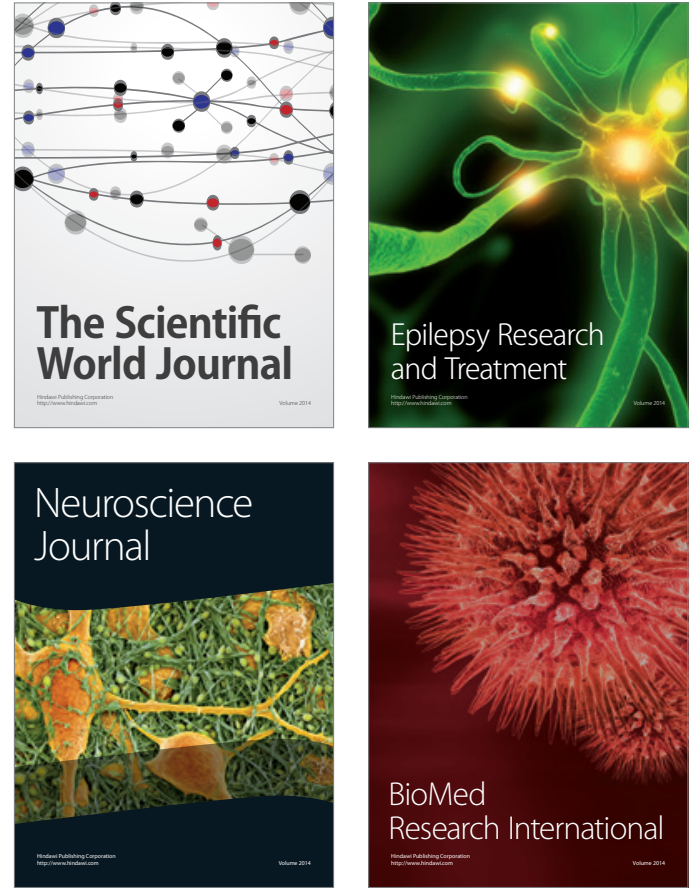

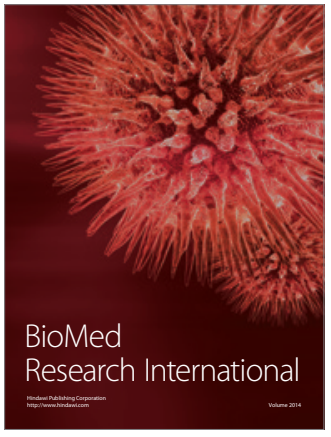

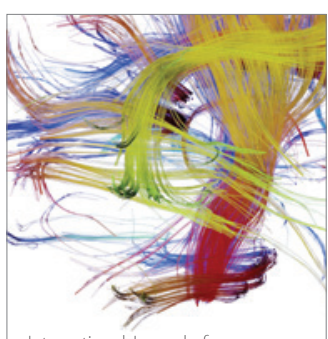

Brain Science

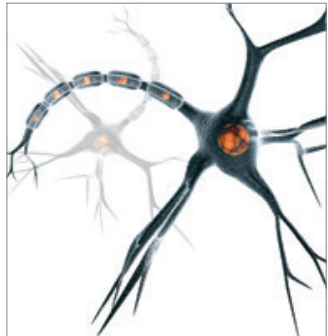

Neural Plasticity
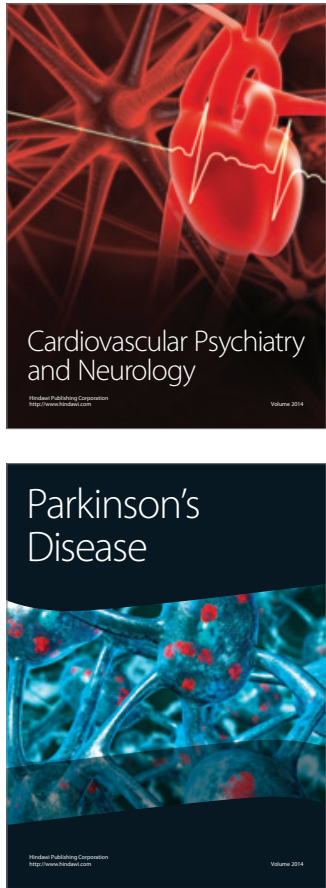